Finisterra, XXXVI, 72, 2001, pp. 195-206

\title{
IDENTIFICAÇÃO DE UNIDADES DE PAISAGEM: Metodologia aplicada a Portugal Continental
}

\author{
T. Pinto-Correia ${ }^{1}$, \\ A. Cancela d’Abreu ${ }^{2}$ \\ R. OLIVEIRA ${ }^{3}$
}

\begin{abstract}
Resumo - Este artigo apresenta os conceitos e a metodologia utilizados no estudo "Identificação e Caracterização da Paisagem em Portugal Continental», elaborado para a DGOT-DU, Ministério do Ambiente e Ordenamento do Território, pelo Departamento de Planeamento Biofísico e Paisagístico da Universidade de Évora, entre 1999 e 2001. A abordagem metodológica adoptada fundamenta-se, por um lado, nas metodologias utilizadas recentemente para estudos semelhantes realizados noutros países da Europa e na forma como a paisagem tem vindo a ser considerada em vários documentos estratégicos ao nível europeu, e por outro lado na preocupação, por parte da equipa, de considerar a paisagem numa perspectiva holística, contemplando as suas várias componentes: ecológica, cultural, socioeconómica e sensorial. Para definir as unidades de paisagem, ao cruzamento da cartografia relativa a variáveis seleccionadas juntam-se assim a análise da imagem de satélite e as observações de campo, mas também uma avaliação por peritagem do caracter e coerência da paisagem. O resultado é uma abordagem flexível, que combina uma análise objectiva com uma outra mais subjectiva, sendo a combinação das duas considerada fundamental pela equipa para a verdadeira apreensão da paisagem.
\end{abstract}

Palavras-chave: Paisagem, unidades de paisagem, apreciação, caracter

Abstract- IDENTIFICATION AND CHARACTERISATION OF LANDSCAPE IN PORTUGAL This paper presents the concepts and methodology used in the study «Identification and characterisation of landscape in continental Portugal» undertaken by the Department of Landscape and Biophysical Planning of the University of Evora for the General Directorate for Spatial Planning and Urban Development (DGOT-DU) at the Ministry of the Environment and Spatial Planning, between 1999 and 2001. On the one hand, the methodological approach developed is based on the methodologies used recently for the same purpose in different European countries and on the way landscape has been considered in various European documents in the last years. On the other hand, it is also based on the team's concern to approach the landscape as an holistic entity, and to examine its various components: ecological, cultural, socio-economic and sensorial. The set aim has been to define landscape units and to characterize these units in relation to the present landscape

1 Professora Auxiliar. Universidade de Évora, Departamento de Planeamento Biofísico. Colégio Luis Vervey-7000 Évora. Tel.266 744 616, Fax. 266744 968, E-mail: mtpc@uevora.pt

2 Professor Associado da Universidade de Évora. E-mail: adabreu@uevora.pt

3 Assistente de Investigação da Universidade de Évora. E-mail: rosario_oliveira@hotmail.com 
and the recorded trends, related problems and possibilities. Thus, the cartography relative to selected variables has been combined and related to satellite images and field surveys. The results of cross-referencing all this information has than been combined with expert examination of landscape coherence and character within each unit. The assessment was completed after careful bibliographic research and consultation with regional experts. The result is a flexible approach that combines objective analysis with a more subjective assessment, which the team considered fundamental for a true understanding of landscape.

Key words: Landscape, landscape units, assessment, character.

\section{INTRODUÇÃO}

$\mathrm{Na}$ Europa, as paisagens têm recentemente vindo a ser reconhecidas como uma componente fundamental do património natural, histórico, cultural e científico. Tal como se defendia já em 1995 no «Dobris Assessment» (STANners e Bourdeau, 1995) e, mais recentemente, na Convenção Europeia da Paisagem (Conselho Da Europa, 2000), as paisagens constituem um elemento fundamental da identidade local e regional, e até, a uma outra escala, europeia. Os objectivos da Convenção partem da constatação de que as paisagens europeias, devido a uma diversidade de factores, se encontram num processo acelerado de transformação em várias e diferentes direcções, o que justifica a necessidade de intervenção. A UNESCO e a IUCN já anteriormente defendiam a protecção de paisagens de elevado valor cultural ou natural mas, a partir dos anos 90, passou a considerar-se a paisagem em todo o território, e todos os tipos de paisagem. A «Estratégia Pan-Europeia para a Diversidade Biológica e de Paisagem» (EcNC, 1996) apresenta a necessidade de integrar a escala da paisagem na perspectiva de conservação da natureza. A sua gestão como base para a integração das componentes sociais e ambientais é considerada no texto da Agenda XXI. Em vários documentos de âmbito europeu, a paisagem, vista como a expressão das numerosas relações estabelecidas ao longo do tempo entre os factores naturais e humanos num determinado território, tem sido considerada como uma das bases mais adequadas para a gestão integrada e equilibrada do espaço europeu (GREEN, 2000; WASHER, 2000; WASHER e Jongman, 2000; Vos e KliJn, 2000). O relatório «Princípios Directores para o Desenvolvimento Espacial Sustentável do Continente Europeu»(CEMAT, 2000) reconhece que a paisagem é o reflexo da diversidade do território europeu, e que por isso deve estar ligada ao ordenamento do território, que organiza os vários sectores em relação ao seu impacto no território.

Tanto estes «Princípios Directores» como a Convenção Europeia da Paisagem (CONSElHo DA EuROPA, 2000), já assinada por vários países, entre os quais Portugal, definem a urgência de criar políticas de paisagem, visando a sua protecção e gestão, e integrando-a em vários outros tipos de política. É para isso necessário identificar as paisagens, os seus limites, o seu carácter, as tendências e ameaças a que estão sujeitas. Só este reconhecimento pode levar à definição 
de estratégias e instrumentos numa lógica de subsidariedade, seguindo orientações mais vastas mas respeitando a especificidade local e mantendo a sua identidade (CEMAT, 2000).

É no âmbito destas solicitações e desta tomada de consciência do papel que a paisagem pode assumir no ordenamento que surge o estudo «Identificação e Caracterização das Paisagens em Portugal Continental», resultado de uma encomenda da Direcção Geral do Ordenamento do Território e Desenvolvimento Urbano, do Ministério do Ambiente e do Ordenamento do Território. Este projecto é acompanhado por um estudo com os mesmos objectivos em Espanha, sendo ambos financiados pelo programa Interreg IIC. O estudo foi desenvolvido na Universidade de Évora, por uma equipa interdisciplinar, apoiada por um grupo de consultores com formações e áreas de interesse muito diversas. A mesma equipa elaborou também um estudo equivalente para os Açores, para a Secretaria Regional do Ambiente da Região Autónoma dos Açores.

O objectivo do estudo a que se refere este artigo é assim o de considerar de uma forma homogénea todo o território continental português e de identificar unidades de paisagem, cada uma com um carácter específico, caracterizando tão detalhadamente quanto possível cada uma dessas unidades, de forma a constituir um apoio à orientação de estratégias e instrumentos de ordenamento ou das políticas sectoriais com maior incidência na paisagem.

\section{A PAISAGEM}

O conceito de paisagem tem sido muito discutido e pode ser considerado de formas variadas por diferentes disciplinas e perspectivas. A abordagem interdisciplinar e holística da ecologia da paisagem, desenvolvida há algumas décadas, veio reforçar e desenvolver o conceito de paisagem como um sistema (BRANDT, 1998). A paisagem é considerada por vários autores como um sistema complexo, dinâmico, onde vários factores naturais e culturais se influenciam mutuamente e se modificam ao longo do tempo, determinando e sendo determinados pela estrutura global (FARINA, 1997; Forman E Godron, 1986; NAVEH E LIEBERMAN, 1994; ZONNEVELD, 1990). A compreensão da paisagem implica, assim, o conhecimento de factores como a litologia, o relevo, a hidrografia, o clima, os solos, a flora e a fauna, a estrutura ecológica, o uso do solo e todas as outras expressões da actividade humana ao longo do tempo, bem como a análise da sua inter-relação, o que resulta numa realidade multifacetada.

Torna-se ainda claro que a esta componente objectiva, composta por uma combinação de factores abióticos e bióticos (suporte físico, meio biológico e acção humana), se acrescenta uma componente subjectiva, que corresponde às impressões causadas por esta combinação em cada observador (FromENT e JoYCE, 1987; SARAIVA, 1999). Um conjunto de autores refere-se à interacção entre o sistema natural e o sistema social, conferindo à paisagem uma dimensão 
territorial e cultural, no sentido em que o modo de apropriação da paisagem pelas comunidades presentes varia tanto com o sistema natural, como com os valores da sociedade que sobre ela actua (ANDRESEN, 1992; BERNALDEZ, 1981; SARAiva, 1999; Telles, 1985). Para Marc Antrop (2000a), o carácter perceptivo está intimamente ligado ao conceito holístico da paisagem e pode ser visto como a síntese necessária à abordagem interdisciplinar da ecologia da paisagem. A introdução da componente subjectiva na análise da paisagem tem no entanto sido pouco testada, talvez por exigir a combinação complexa de metodologias diversas e o desenvolvimento de novos instrumentos de avaliação.

A noção do carácter da paisagem tem vindo a ser introduzida neste debate, como uma componente fundamental para a sua compreensão de uma forma integrada e considerando todas as componentes. O relatório «European Landscapes», elaborado para a Agência Europeia do Ambiente (WASHER E JongmAN, 2000), afirma que «quer seja à escala local, regional, nacional, ou ainda internacional, as paisagens exprimem a unicidade e identidade de cada lugar (genius loci), reflectindo tanto a história natural como cultural de um território, num determinado momento». O carácter é dinâmico e está continuamente em mudança, mas é único para cada lugar, e tem um papel preponderante no estabelecimento da identidade local (ANTrop, 2000b; Hughes E Buchan, 1999; WASHER, 1999). O papel da paisagem na identidade local e regional tinha já sido destacado por Orlando Ribeiro, ao afirmar que a paisagem de hoje, correspondendo a um produto do passado, constitui um registo da memória colectiva (Ribeiro, 1993). Tal como salienta Jorge Gaspar (1993), «a paisagem torna-se um elemento tão poderoso de identificação cultural que, como a língua e a religião - no que ela transporta de código comportamental-entra no pano de fundo do universo onírico (...) E o mais espantoso é que, ainda como a língua e a religião, também a paisagem se actualiza permanentemente».

Na língua francesa, o "pays» - com ligações claras ao termo "paysage» exprime de forma clara esta identificação: o "pays é um território, com uma paisagem que lhe é própria, com características naturais, sociais e culturais suficientemente homogéneas para contribuírem para a existência e reconhecimento da sua identidade, quer pelos que lá vivem como pelos que o consideram do exterior (JANIN, 1995).

Estes conceitos referem-se sempre a uma paisagem cultural, dominante no espaço europeu, expressão dos diversos factores naturais existentes mas, também, da acção humana sobre esses factores. A paisagem natural seria aquela em que a articulação dos diversos factores naturais ao longo do tempo não fosse afectada pela acção humana, o que só acontece muito pontualmente na Europa. Caldeira Cabral et al 1978, estabelece uma distinção básica entre paisagem natural - «resultado da interacção exclusiva dos factores físicos e bióticos, anteriores à acção do Homem», acrescentando que se trata «apenas de um conceito lógico, sem existência no mundo»-e paisagem humanizada "resultante da acção multissecular, contínua ou intermitente, do homem sobre a paisagem 
natural, apropriando-a e modificando-a a fim de a adaptar pouco a pouco às suas necessidades, segundo o que a sua experiência, os seus conhecimentos e a sua intuição lhe foram ensinando, experiência transmitida de geração em geração".

Com o objectivo da compreensão total da paisagem, o estudo aqui apresentado procura ser holístico e integrador das várias componentes: a ecológica, relativa à parte física e biológica dos ecossistemas; a cultural, onde tanto os factores históricos como as questões de identidade e as qualidades narrativas da paisagem são considerados; a socio-económica, referindo-se aos factores sociais e às actividades económicas, assim como as respectivas regulamentações, condicionadoras da acção humana que permanentemente constrói e transforma a paisagem; e finalmente a sensorial, ligada às impressóes causadas pela paisagem. A paisagem é considerada neste estudo como um sistema dinâmico, onde os diferentes factores naturais e culturais se influenciam entre si e evoluem em conjunto, determinando e sendo determinados pela estrutura global, o que resulta numa configuração particular de relevo, coberto vegetal, uso do solo e povoamento, que lhe confere uma certa coerência e à qual corresponde um determinado carácter.

Combinando as várias componentes acima mencionadas, as unidades de paisagem identificadas em Portugal Continental correspondem a áreas com características relativamente homogéneas no seu interior, não por serem exactamente iguais em toda a área, mas por terem um padrão específico que se repete e que diferencia a unidade em causa das envolventes. Os factores determinantes para a especificidade da paisagem numa unidade não são sempre os mesmos: podem ser as formas do relevo, a altitude, o uso do solo, a urbanização, várias combinações entre estes factores, etc. Para além do padrão de paisagem específico, considerou-se que devia existir uma coerência interna e um carácter próprio em cada unidade, identificável do interior e do exterior, e eventualmente associado às representações da paisagem mais fortes na identidade local e/ou regional. Esta definição de unidade de paisagem corresponde em traços largos ao conceito de "landscape character area», utilizado pelos ingleses e escoceses (COUNTRYside COMMISSION, 1998; Usher, 1999); estas unidades podem ser identificadas através de métodos quantitativos, de cruzamento de múltiplas variáveis, mas implicam também uma aferição por especialistas em paisagem que possam confirmar e descrever o seu carácter. O mesmo tipo de conceito de unidade de paisagem foi utilizado no estudo norueguês e no finlandês, onde se parte de regiões com características naturais homogéneas, para a definição, dentro destas, de unidades de paisagem correspondendo a uma coerência interna e um carácter próprio, identificável do interior e do exterior, associado às características culturais (FRY et al.,1999; MANSIKKA et al., 1993; Pushman, 1998).

As unidades são definidas a uma escala de base uniforme, com a qual se aborda todo o território continental. A uma escala maior seriam normalmente 
identificadas outras unidades, ou sub-unidades, nas quais aumentaria a homogeneidade. A subdivisão em vários níveis hierárquicos é possível, tal como demonstra o estudo de identificação das paisagens da Eslovénia (MARUsiK et al., 1998), e essa opção depende obviamente dos objectivos e recursos do estudo.

Quanto aos elementos da paisagem, o conceito a eles associado varia também com a perspectiva e, sobretudo, a escala de abordagem, sendo também designados de formas bastante variadas. Neste estudo os elementos da paisagem são considerados como aqueles cujo conjunto define a estrutura da paisagem, e cuja identificação permite a análise da paisagem a uma escala de pormenor. São tomados em conta aqueles que se distinguem na fotografia aérea, tanto de origem natural como antrópica. São assim elementos da paisagem, por exemplo, os grandes afloramentos rochosos, as linhas de água, as galerias ripícolas, as sebes de compartimentação e os muros de pedra solta, os alinhamentos de árvores, os bosquetes e as matas, as parcelas cultivadas, e também os conjuntos edificados, como assentos de lavoura, aldeias e áreas urbanas, estradas ou autoestradas, barragens e as respectivas albufeiras, etc.

\section{METODOLOGIA}

De forma a assegurar a combinação de perspectivas e de componentes mencionada acima, o desenvolvimento da metodologia desenvolvida no estudo «Identificação e Caracterização das paisagens em Portugal Continental» assentou, num primeiro momento, na composição duma equipa interdisciplinar, integrando maioritariamente arquitectos paisagistas e geógrafos, com várias especialidades e experiências de trabalho. A equipa foi também apoiada por um grupo diversificado de consultores, provenientes de várias disciplinas.

A definição da metodologia baseou-se na consulta pormenorizada dos vários estudos actualmente em curso ou já terminados em países europeus, e no contacto com alguns dos especialistas que trabalharam nestes estudos, tendo sido de seguida definida uma abordagem adequada aos objectivos nacionais, à informação existente, e aplicável no âmbito do tempo e recursos do projecto.

Para o enquadramento do estudo, é de salientar uma fase importante de revisão da bibliografia existente quanto à caracterização do território português, com diferentes visões e por diferentes especialistas.

Para a definição das unidades de paisagem em todo o território, podem distinguir-se duas fases complementares:

1) Trabalho de gabinete, composto por:

- Selecção de variáveis a considerar explicitamente. Foram consideradas a litologia, a morfologia, a hidrografia, os solos, o uso do solo, a estrutura da propriedade, e o povoamento. Outras variáveis fundamentais, como o 
clima ou a proximidade do oceano, foram consideradas implicitamente. A escala de trabalho adoptada foi 1:250 000 .

- Recolha da informação disponível para todo o país, para cada uma daquelas variáveis e, quando necessário, tratamento cartográfico dessa informação para todo o território. Recolha de informação complementar, tal como imagens de satélite e fotografia aérea (ortofotomapas). Pretendeu-se que toda a informação estivesse em formato digital, de forma a ser tratada num Sistema de Informação Geográfica, utilizando o programa ArcView.

- Cruzamento da informação relativa às variáveis consideradas, ponderado pelo conhecimento dos membros da equipa e pela informação recolhida através da bibliografia sobre as paisagens portuguesas. Deste cruzamento resultou um primeiro esboço de delimitação de unidades de paisagem.

- Verificação deste primeiro esboço, com base no conhecimento dos vários membros da equipa, nos percursos de campo efectuados e, principalmente, nas imagens de satélite e nos ortofotomapas (expressão sintética de padrões paisagísticos diferenciados). Em simultâneo, primeira aproximação à designação das unidades, de forma a obter denominações coerentes mas também facilmente reconhecíveis por técnicos e pelo público.

2) Trabalho de campo, composto por:

- Verificação da coerência e dos limites definidos no esboço de gabinete, através de percursos no terreno, segundo trajectos previamente definidos, de forma a cobrir todas as unidades, e sobretudo as áreas menos conhecidas pela equipa e onde maiores dúvidas tinham sido detectadas.

- Apresentação das unidades a interlocutores privilegiados, seleccionados previamente, de forma a recolher informação e opiniões quanto à coerência das unidades e dos seus limites, ao seu carácter e à sua designação.

Esta fase foi ainda seguida por outro momento de verificação em gabinete que envolveu grande parte da equipa, com o objectivo de, mais uma vez, verificar a coerência e os limites das unidades e introduzir as correcções necessárias. Tais correcções continuaram à medida que se foram preenchendo as fichas relativas a cada unidade de paisagem.

A delimitação das unidades mostrou ser uma tarefa complexa, uma vez que raramente a transição de uma unidade de paisagem para uma outra se faz através de uma linha de mudança brusca. O carácter de cada unidade de paisagem, reflectido num padrão que lhe é característico é, na maioria dos casos, muito claramente definido numa área central da unidade, constituindo 
uma espécie de núcleo. Entre as áreas nucleares de unidades adjacentes, há um espaço de transição mais ou menos extenso. Nalguns casos estas características de transição atingem tais dimensões que justificam a individualização de uma unidade, cujo carácter é pouco definido e que conjuga aspectos de várias outras unidades próximas. De qualquer modo, os limites estabelecidos não devem ser considerados como linhas claramente definidas e perceptíveis no terreno, mas sim como faixas de transição progressiva. Em certos casos, no entanto, os limites são muito bem definidos, sobretudo quando correspondem a descontinuidades bruscas no relevo ou na natureza das formações litológicas.

O procedimento descrito é baseado no conhecimento e capacidade de síntese dos peritos, e envolve uma real flexibilidade na selecção do ou dos parâmetros que são determinantes em cada situação para a individualização de uma unidade de paisagem. A paisagem mais objectiva, «material», é assim considerada em simultâneo com as suas componentes subjectivas o que, em conjunto, determina o seu carácter.

A caracterização das unidades foi feita fundamentalmente em gabinete, com base na cartografia utilizada durante todo o processo de identificação e na bibliografia seleccionada, sendo esta informação substancialmente enriquecida pelas notas e imagens fotográficas recolhida no campo, tanto nos trajectos efectuados como no contacto com os interlocutores privilegiados. Foram ainda recolhidos vários tipos de dados por concelho ou freguesia (demografia, infraestruturas, áreas recentemente florestadas, áreas ardidas, áreas beneficiadas por medidas agro-ambientais, etc.), com o objectivo de identificar as actuais dinâmicas e tendências de mudança no padrão de paisagem de cada unidade. Esta caracterização foi organizada em fichas por unidade, contendo cartografia, fotografias específicas, e texto: dados relativos à localização geográfica, descrição do carácter da paisagem, informação quanto a características particulares e também quanto ao ordenamento, diagnóstico e orientações para a gestão.

Para além das unidades, foram ainda definidas, sub-unidades de paisagem, áreas que fazem parte da unidade e da sua coerência interna, mas têm características específicas quanto a pelo menos uma variável (morfologia, altitude, presença de água, uso do solo, ocupação urbana, etc.). Nalguns casos constituem-se como paisagens claramente diferentes, mas com dimensão que não justifica a existência de uma unidade separada (por exemplo, a presença de serras com dimensões relativamente pouco importantes, que são consideradas como sub-unidade de um conjunto mais vasto, no geral plano e de altitude mais baixa). Em muitas unidades não se identificou nenhuma sub-unidade, enquanto noutras se identificaram várias sub-unidades.

Foram também individualizados «Elementos Singulares», elementos com reduzida dimensão em termos de superfície ocupada, mas que se destacam no conjunto da unidade de paisagem pela sua diferença, pela qualidade intrínseca (ou, pelo contrário, por constituir uma dissonância desqualificadora) e/ou pelo impacto (sensitivo, cultural ou ecológico) que têm sobre a unidade: elevações ou cabos proeminentes, construções ou conjuntos edificados de elevado interesse 
e relevância em termos de paisagem, ou que se encontram claramente não integrados relativamente à sua envolvente, áreas com uma degradação específica (feridas provocadas por exploração de inertes, vias de comunicação que rasgam a paisagem sem qualquer respeito pelas preexistências, etc.). Exemplos destes elementos singulares podem ser pontos de vista excepcionais, elementos notáveis quanto a vegetação, geologia ou outros valores naturais, grandes infraestruturas, alguns aglomerados urbanos sobretudo pela sua localização, etc.

\section{RESULTADOS}

O trabalho desenvolvido resultou na identificação de 128 unidades de paisagem para Portugal Continental (Fig.1). A definição destas unidades deve ser considerada em relação à escala de análise, 1:250 000. Estas 128 unidades foram organizadas em 22 grupos de unidades, juntando unidades com características semelhantes em relação a um conjunto de características físicas, sobretudo, mas também com traços comuns quanto à ocupação humana.

\section{DISCUSSÃO}

Esta identificação e caracterização de paisagens corresponde a um contexto temporal bem definido. Tendo em conta a dinâmica própria da paisagem, uma aproximação semelhante a este tema desenvolvida num outro momento teria, ou terá, resultados diferentes.

As unidades de paisagem obtidas resultam dos critérios desenvolvidos pela equipa do estudo, baseada fundamentalmente em peritagem pela mesma equipa, pelo que se assume uma relativa subjectividade. As principais fragilidades do método têm a ver com a dificuldade em garantir a homogeneidade no tratamento de todo o território continental, não devido às bases de informação utilizadas mas, principalmente, pelo conhecimento diferenciado deste território por parte dos membros da equipa. Mas a introdução da subjectividade foi desde o início considerada como fundamental para a interpretação do carácter da paisagem. A articulação entre informação objectiva e um julgamento mais subjectivo por peritos, é considerada como uma forte potencialidade desta abordagem.

A metodologia seguida é flexível, adaptável aos mais variados tipos de paisagem, podendo considerar diferentes factores com pesos diversos consoante as situações presentes. Flexível também porque permite uma utilização a vários níveis, podendo aprofundar-se mais ou menos a análise, tanto cartográfica como baseada na recolha de informação de outras fontes. Permite também a continuidade do estudo em várias direcções, quer para aprofundamento de temas determinados, quer para a verificação e melhor caracterização das unidades. 
Fig. 1 - Unidades de paisagem e grupos regionais de unidades Fig. 1 -Landscape units and regional groups of units 
As unidades de paisagem definidas têm coerência interna e individualizamse por um determinado carácter, reconhecido pela equipa e descrito em pormenor nas respectivas fichas. No entanto, tal definição não pode ser considerada duma forma estática, nem como a única possível. Constitui mais uma base de reflexão para a compreensão e conhecimento das paisagens em Portugal Continental, deixando em aberto várias pistas para aprofundamentos futuros.

\section{BIBLIOGRAFIA}

Andersen, M. T. (1992) - Para a Crítica da Paisagem. Dissertação de doutoramento, Universidade de Aveiro.

Antrop, M. (2000a) - Background concepts for integrated landscape analysis. in Agriculture, Ecosystems and the Enviroment 77:17-28.

Antrop, M. (2000b) - Where are the Genii Loci ? in: PEDROLI B. (Ed.), Landscape-Our Home. Indigo Zeist, Stuttgart: 29-34

Bernaldez F.G. (1981) - Ecologia y Paisage. H.Blume Ediciones, Madrid.

Brandt, J. (1998) - New Perspectives for Landscape Ecology. In: Cancela d'Abreu M. E PintoCorreia T. (Eds.), Challenges for Mediterranean Landscape Ecology: the Future of Cultural landscapes-examples from the Alentejo region, Actas do I Encontro Nacional de Ecologia da Paisagem, Montemor-o-Novo.

Caldeira Cabral, F.; Gonçalves, J.E.; Cabral, J.C. (1978) - Ordenamento da Paisagem Rural. Ensaio de Sistematização. DGPU, Lisboa (dact.).

Cemat, (2000) - Guiding principles for Sustainable Spatial Development of the European Continent. European Conference of Ministers responsible for Regional Planning, Council of Europe, Estrasburgo.

Conselho da Europa, (2000) - Convention Européenne du paysage et Rapport explicatif. T-Land (2000)6. Estrasburgo.

Countryside Commission, (1998) - Countryside Character. The character of England's natural and man-made landscape. Countryside Commission, Walgrave. Northampton.

Ecnc, (1996) - The Pan-European Biological and Landscape Diversity Strategy. Council of Europe, UNEP, European Centre for Nature Conservation, Tilburg, The Netherlands.

FARINA, A. (1997) - Principles and methods in landscape ecology. Chapman and Hall, London.

Forman, R.; Godron, M. (1986) - Landscape Ecology. John Wiley \& Sons, New York.

Froment, A.; Joye CH. (1987) - La paysage, patrimoine naturel et culturel, Example du Pays de l'Herve. Notes de Recherche de la Societé Géographique de Liège, 8: 37-48.

Fry, G.; Pushmann, O.; Dramstad, W. (1999). Geographic Information for Research and Policy. A Norwegian Landscape Perspective. In USHER M.B.(Editor), Landscape Character. Perspectives on Management and Change. The Stationery Office. Scottish Natural Heritage and Macaulay Land Use Research Institute, Edinburgh: 189-203.

Gaspar, J. (1993) - As Regiões Portuguesas. Direcção Geral do Desenvolvimento Regional, Lisboa. 
Green, B.H. (2000) - Policy, planning and management initiatives in European cultural landscape conservation. In: KLIJN J. and VOS W. (Eds), From Landscape Ecology to Landscape Science. Kluwer Academic Publishers. Dordrecht, Boston and London: 57-72.

Hughes, R.; Buchan, N. (1999). The Landscape Character Assessment of Scotland. In: USHER M.B.(Editor), Landscape Character. Perspectives on Management and Change. The Stationery Office. Scottish Natural Heritage and Macaulay Land Use Research Institute, Edinburgh: 1-12.

JANIN, C. (1995) - Peut-on «faire l'économie» du paysage pour gérer le territoire ? L'agriculture dans le paysage, une autre manière de faire du développement local. Dossier de la Revue de Geographie Alpine, 15: 11-30.

Makhzoumi, J.; Pungetti G., (1999) - Ecological Landscape Design and Planning. The Mediterranean Context. E \& FN Spon, London.

Mansikka, M.; Heikkila, T.; Strandel, C. (1993)-National Landscapes. Ministry of the Environment, Finland, Helsinki.

Marusic J. et al, (1998) - Regional Distribution of Landscape Types in Slovenia. Methodological Bases, National Office for Physical Planning, Republic of Slovenia.

Naveh Z.; Lieberman A. (1994) - Landscape Ecology - Theory and Application. Spinger-Verlag, New York.

Pushmann, O. (1998)-The Norwegian landscape reference system-use of different sources as a base to describe landscape regions. NIJOS Report 12/98, Norwegian Institute of Land Inventory, Aas, Norway.

Ramos, I. (1998) - A paisagem da Ribeira das Alcáçovas: uma abordagem socio-ecológica. Disseratção de Mestrado, Faculdade de Letras da Universidade de Lisboa, Lisboa.

Ribeiro, O. (1993) - Portugal, o Mediterrâneo e o Atlântico. Sá da Costa, Lisboa.

SARAIVA, G. (1999) - O Rio como paisagem. Gestão de corredores fluviais no quadro do ordenamento do território. Fundação Calouste Gulbenkian e Fundação para a Ciência e Tecnologia, Lisboa.

Stanners, D.; Bourdeau, P. (Eds.), (1995)-Europe's Environment. The Dobris Assessment: an overview. European Union, Luxembourg.

Telles, G.R. (1985) - Para além da Revolução. Edições Salamandra, Lisboa.

Usher, M. B. (Editor), (1999). Landscape Character. Perspectives on Management and Change. The Stationery Office, Scottish Natural Heritage and Macaulay Land Use Research Institute, Edinburgh.

Vos, W.; KLIJN, J.K. (2000) - Trends in European landscape development: prospects for a sustainable future. In: KLIJN J. and VOS W. (Eds), From Landscape Ecology to Landscape Science.. Kluwer Academic Publishers. Dordrecht, Boston and London: 13-29.

WASHER, D. (Ed.), (1999) - Landscapes and Sustainabilty. Strasbourg.

WAsher, D. (Ed.), (2000) - The Face of Europe. Policy Perspectives for European Landscapes. ECNC Technical Report Series. European Centre for Nature Conservation, Tilburg.

Washer, D.; Jongman, R. (Eds.), (2000)-European landscapes. Classification, assessment and conservation. Draft -. European Environmental Agency, Copenhagen.

ZonNEVELD, I.S. (1990) - Scope and concepts of Landscape Ecology as an emerging science. In: Forman F. and Zonneveld I.S. (Editors), Changing Landscapes: An Ecological Perspective, Spinger-Verlag, New York. 